\title{
Cumplimiento institucional en la defensa de los derechos humanos a partir de los acuerdos de paz
}

\author{
René Edgardo Vargas Valdez \\ crene.vargas@utec.edu.sv \\ Investigador UTEC
}

\section{Resumen}

El Acuerdo de paz firmado entre el Gobierno de El Salvador y la insurgencia aglutinada en el Frente Farabundo Martí para la Liberación Nacional (FMLN) constituyó un acontecimiento de resonancia a escala mundial y un ejemplo de lo que se puede lograr con voluntad política; sin embargo, el haber hecho renacer en la población el sueño de una sociedad más justa, equitativa y encaminada hacia la búsqueda del bien común, pronto se fue convirtiendo en desencanto ante una realidad que hacía cada vez más precaria la vida de una gran mayoría de la población, de ahí nuestra hipótesis de trabajo "Las instituciones responsables de la defensa de los derechos humanos han adolecido de unas creativas y claras políticas públicas para poder cumplir sus mandatos", la cual hemos tratado de demostrar mediante un análisis basado en la integración sistemática de diferentes estudios sobre el tema, interpretados a través del marco teórico general de la Filosofía Política y el método comparativo en los aspectos jurídicos, empleando los ejes temáticos del modelo de evaluación institucional elaborado por el Observatorio de la Democracia en Centroamérica.

Luego de 20 años, la contradicción entre el compromiso formal de cumplir lo mandatado en la Constitución y el modelo impulsado, ha legado una crítica situación económica y social, con la consecuente crisis de gobernabilidad asumida por un gobierno de izquierda, suceso sin presedentes en el país dentro de su larga historia política.

Palabras clave: el signo de los tiempos. Criterio de verdad. Bien común. Derechos humanos de primera y segunda generación. 


\section{ABSTRACT}

The peace agreement signed between the Government of El Salvador and the bonded insurgency in the Frente Farabundo Marti para la Liberacion Nacional (FMLN) was an event of global resonance and an example of what can be achieved with political will, but , having made reborn the people's dream of a more just, equitable society aimed towards the search for the common good, it soon grew into disillusionment with a reality that was making life increasingly precarious for the great majority of the population, hence our working hypothesis "The institutions responsible for defending human rights have lacked from creative and clear public policies to meet its mandates," which we have tried to demonstrate through an analysis based on the systematic integration of different studies on the subject, read through the general framework of political philosophy and the comparative method in legal issues, using themes of institutional assessment model developed by the Centre for Democracy in Central America.

After 20 years, the contradiction between the formal commitment to implement the mandated in the constitution and the impulse model, left us a critical economic and social situation, with the consequent crisis of governance taken by a left aligned government, an event unprecedented in the country in his long political history.

Keywords: The sign of the times. Criterion of truth. Common good. Human rights of first and second generation.

\section{INTRODUCCIÓN}

El advenimiento del siglo XX avivó excepcionales emociones para la humanidad por los avances tecnológicos y científicos que le precedieron, así como por la consolidación de las transformaciones políticas y sociales iniciadas a partir de los siglos XVIII y XIX. Al arribar al clímax del segundo milenio, las percepciones eran agobiantes dadas las experiencias vividas al final del camino e inicio de un desconocido devenir.

Adentrados casi un decenio después, el entorno en el que los diferentes actores externos e internos estamos viviendo el presente, ha puesto a prueba nuestra creatividad en el esfuerzo por parir nuevas alternativas políticas y económicas con el fin de superar la brecha entre el deber ser y la acuciante realidad; esa es la coyuntura resultante de una serie acumulada de fenómenos convertidos en una situación estructural que requiere ser comprendida y explicada desde su génesis histórica, por cuanto sobre ella hemos de construir el futuro. Paradójicamente, lo que se nos presenta como una visión caótica ha generado su propio contrario: la posibilidad del orden¹. El concretarlo dependerá de la reelaboración que realicemos sobre sus causas. Es difícil lograr una visión completamente objetiva acerca de los hechos estudiados, por ello resulta legítimo considerar que siempre habrá en toda investigación social una dosis de subjetividad, lo cual es saludable en la medida en que se quede al nivel de identificación con una forma de

\footnotetext{
${ }^{1}$ Contrario al dogma del orden universal, en donde el permanente equilibrio fue, es y será lo fundamental, la ciencia ha demostrado que el caos es constitutivo de la naturaleza, de la sociedad y del universo, existiendo un desorden armonioso necesario para la continuidad. Siendo un nuevo paradigma para concebir al mundo, ofrece al investigador social sugerentes ámbitos de estudio, en especial acerca de esos factores subjetivos, impredecibles e inciertos, para comprender sus efectos en el orden social, en donde el caos se encuentra siempre presente en mayor o menor medida.
} 
pensamiento, pero sin perder el sentido de racionalidad que permite desarrollar el conocimiento científico, resurgido con el legado intelectual y político del Renacimiento, de la Ilustración y de la Revolución francesa, movimientos cuyo espíritu de su época fueron el saber y el principio de la libertad. Para alcanzar esa adecuada relación entre el pensamiento y la razón, la filosofía nos proporciona una serie de categorías a emplear, que parten de aceptar la existencia de una conciencia individual, como también de una cognición colectiva constituida por los elementos comunes entre las distintas formas de pensamiento particular expresadas socialmente a través de normas 0 modelos de comportamiento aceptados por la mayoría. En estas aportaciones se sustenta la discusión sobre la solución metafísica del idealismo y materialista del realismo, como esencias del conocimiento a consecuencia de la dualidad inherente al hombre,ser de acción y de conciencia.

La desconfianza, el miedo y la frustración, son actitudes que responden a las condicionantes externas sobre el diario vivir de la gran mayoría de los pobladores en el mundo actual, cuyo impacto es mucho mayor que su propia capacidad interna para enfrentarlos, sobre todo cuando sus energías son absorbidas en el esfuerzo de la lucha por sobrevivir. Según un reciente informe del Programa de las Naciones Unidas para el Desarrollo (PNUD, 2010) sobre desarrollo humano, 1.4 millones de personas viven con solo un poco más de un dólar al día y en 2005 la población en nivel de pobreza alcanzaba la cifra de 984 millones; en relación con América Latina y el Caribe, el estudio de la Cepal del 2008 menciona que aproximadamente 182 millones de personas vivían en la pobreza, afirmando que era la región más desigual del mundo, situación que constituye un grave obstáculo para su desarrollo y ha provocado muy exiguas posibilidades de movilidad social.
Explicar esta realidad en El Salvador actual es el objetivo general de nuestro estudio, bajo el título "Cumplimiento institucional en la defensa de los derechos humanos a partir de los Acuerdos de Paz". Se trata de un esfuerzo académico por superar el relato histórico-cronológico y enciclopedista, e intentar una interpretación metódica de los hechos desde un enfoque jurídico-sociológico y filosófico político, con la finalidad específica de aportar alternativas de solución a los problemas que enfrentamos ante la crisis mundial e interna, primordialmente en lo que atañe a la gobernabilidad democrática y sus repercusiones en el respeto de los derechos humanos, sobre la base de las hipótesis general y de trabajo siguientes: “La intervención de los factores reales de poder en las decisiones del Estado han atenuado el logro de los objetivos de sus instituciones y atentado en contra de la gobernabilidad" y "Las instituciones responsables de la defensa de los derechos humanos han adolecido de unas creativas y claras políticas públicas para poder cumplir sus mandatos".

\section{METOdOLOGÍA}

Siendo nuestro objeto de estudio el acuerdo de paz en lo referido a los derechos humanos, constituye su espacio temporal la interpretación de los acontecimientos acaecidos a partir de la firma del pacto en 1992, hasta la última presidencia del partido Alianza Republicana Nacionalista (Arena), que concluyó en mayo de 2009; por otra parte, se ha segmentado la investigación partiendo de lo general a lo particular, ubicando primeramente al país dentro del proceso de transición que vive actualmente el mundo, continuando luego con el esfuerzo político interno por cambiar el rumbo en la búsqueda de un destino mejor y establecer posteriormente el estado de la gobernabilidad democrática dentro del período estudiado, así como la situación de la 
aplicación de justicia y de los derechos de primera y segunda generación. Finalmente analizamos de manera cuantitativa y cualitativa la evolución de ambas formas de derechos mediante el método comparativo, recopilando información de otros estudios y encuestas de opinión, así como de algunas entrevistas previamente estructuradas, con el fin de conocer diversos puntos de vista sobre situaciones específicas a interpretar, tratando de respondernos las siguientes interrogantes: ¿Han sido cumplidos los objetivos propuestos en el acuerdo de paz? ¿Existe una reafirmación del Estado de derecho? ¿Han cumplido las instituciones surgidas del acuerdo las funciones para las que fueron creadas?

\section{ANÁLISIS Y DISCUSIÓN DE RESULTADOS}

Es innegable que se han dado algunos cambios positivos, reales y formales, en la sociedad salvadoreña. Resulta esencial la mejora institucional como un factor importante en la determinación del tipo y calidad de la democracia pensada; en ese sentido, merece una mención especial el que el FMLN haya reconocido la evolución de la Fuerza Armada después del acuerdo de paz. Otro elemento por señalar es el desarrollo de la estructura del Estado para posibilitar el cumplimiento de los acuerdos, instaurando organizaciones como:

- La Comisión Nacional para la Consolidación de la Paz (Copaz)

- La Policía Nacional Civil (PNC), en sustitución de los cuerpos de seguridad,

- La Procuraduría para la Defensa de los Derechos Humanos (Pddh)

\section{- El Tribunal Supremo Electoral (TSE)}

También deben ser incluidos los cambios formalesde reformas constitucionales para mejorar aspectos del sistema judicial, referidos a una manera diferente de organizar y elegir a los Magistrados de la Corte Suprema de Justicia (CSJ), la elección del Fiscal General de la República, del Procurador General de la República y del Procurador para la Defensa de Ios Derechos Humanos, así como la redefinición estructural del Consejo Nacional de la Judicatura (CNJ).

Pero el Estado de derecho se ha visto permanentemente amenazado por la poca cultura del respeto a la norma jurídica, convirtiendo la convivencia social entre los salvadoreños en una lucha del "sálvese quien pueda", la libertad en libertinaje, y recurriendo al mal interpretado principio de que "el fin justifica los medios". Desde el acuerdo de paz han existido muchos hechos para demostrar lo afirmado; citamos solo tres de ellos dentro de la pródiga historia del quehacer institucional: la ley de salvataje de los partidos políticos, la tardanza en la aplicación de justicia en graves casos de corrupción, así como en las resoluciones de varios sumarios por recursos de inconstitucionalidad, y el entrampamiento en la aprobación de la ley de medicamentos.

Este es el escenario jurídico-político en el cual nace y evoluciona la PDDH, sensu stricto, las reformas constitucionales crearon las condiciones para la modernización del Estado formal de derecho, sin que por ello se haya logrado su firmeza y consolidación para posibilitar un sistema de gobernabilidad democrática cumplidor de los derechos civiles, políticos, económicos, sociales y culturales de la población, ni que viabilizaran el desarrollo y la anhelada justicia social. El perfil anterior contrapone la visión de quienes justiprecian nuestro Estado de derecho en 
su funcionalidad, partiendo de una evaluación sustentada sobre el derecho positivo, con la constitución real y teleológica, o sea, con esa vasta problemática implicada en la convivencia social que pretende alcanzar relaciones de armonía e integración.

La categoría Derechos Humanos establece una relación intrínseca con el mundo del derecho en el ámbito de cada Estado y con los organismos internacionales que los proclaman; esta interacción trasciende a lo humano, es decir, a las diferentes situaciones históricas y sociales en la evolución del hombre y las concepciones elaboradas acerca de él.

Desde ambas perspectivas surgen diversos criterios para teorizar sobre el tema, ya se trate de obligaciones o compromisos formales desde lo jurídico, o de aquellos derechos considerados humanos por naturaleza y/o los adquiridos a través de un proceso evolutivo; pero sí es un discernimiento aceptado en el ámbito jurídico-político que los derechos fundamentales reconocidos por cada Estado persiguen una finalidad común con los tratados internacionales, a saber: realizar la concepción humanista del Estado y de la sociedad. Ahora bien, el acuerdo de paz se situó de forma preeminente en la exigencia de cumplir con los derechos civiles y políticos o de primera generación, formalmente reconocidos en la Constitución de 1983 en su título II, capítulo I, artículos del.2 al 28,partiendo del principio ético básico de su razón de ser: la libertad (Art4). Las situaciones imperantes en cuanto al irrespeto a la vida, a la integridad física y al pensamiento del contrario, basadas en la tesis de "estás conmigo o estás contra mí", condicionaron la relevancia dada a dicho enfoque, e incluso, la gravedad de la situación forzó a las partes a constituirlo en el primer compromiso parcial entre el gobierno y la insurgencia, firmado el 26 de julio de 1990 en San José, capital de Costa Rica. No obstante, los derechos económicos, sociales y culturales, denominados de segunda generación, ${ }^{2}$ también reconocidos por nuestro marco jurídico constitucional en el capítulo II, en los Artículos.32 al 70, fueron pactados en el capítulo 5 del Acuerdo final para ser tratados en el tema económico social, quedando establecido su estudio y definición mediante la creación del "Foro para la Concertación Económica y Social."

Hemos de reconocer el avance en los derechos humanos de primera generación, pero persiste la deuda histórica de decirle al pueblo la verdad sobre lo acontecido en los años 80. Sobre este tema se entrevistó a diferentes sectores, formulándoles la pregunta ¿cuál consideran sea la mejor alternativa para que el Estado pueda cumplir con la trilogía verdad-justicia-perdón ante las violaciones a los derechos humanos ocurridos entre 1980 y 1991? La mayoría coincidió en que la acción debe ser política y no jurídica, afirmando que es posible encontrar opciones, pues se trata de violaciones a los derechos humanos y al derecho humanitario, cuyas doctrinas han evolucionado dinámicamente, pero se requiere de una institucionalidad estatal fortalecida, condición que por ahora no se cumple en El Salvador, por consiguiente, consolidarla es la primera acción gubernamental para avanzar en la reconciliación de la sociedad.

El fundamento filosófico-político de los denominados derechos de segunda generación deviene de establecer

\footnotetext{
${ }^{2}$ Estos derechos se desarrollaron a finales del S.XIX e inicios del XX, siendo incorporados en la "Declaración Universal de los Derechos Humanos" elaborada en 1948 por la ONU; constituyó un salto de calidad en la configuración del Estado de derecho basado en la libertad individual, al pasar a convertirse en un Estado social de derecho, sustentado en el principio de la solidaridad. El constitucionalismo también adquiere la característica social al establecer la exigencia al Estado de implantar programas y acciones a fin de lograr que la población goce de los derechos económicos, sociales y culturales.
} 
que, para darle plena vigencia a la dignidad humana, a la libertad y a la democracia es condición sine qua non el que existan las condiciones económicas, sociales y culturales que abonen al desarrollo de los pueblos; los negociadores de la paz sabían de la dimensión del reto para establecer el modelo adecuado como complemento al diseño político, desde el cual iniciáramos la ruta de la refundación del Estado de derecho en una sociedad capaz de garantizar la equidad, la diversidad, la individualidad y la sociabilidad, estas últimas como condiciones inherentes a todo ser humano. Por eso, en el capítulo 5, referido al "tema Económico Social" se afirmaba el incluir solo una "plataforma mínima de compromisos tendientes a facilitar el desarrollo en beneficio de todos los estratos de la población."Por esa misma razón, la investigación solo traza unas pinceladas sobre este aspecto, pues evaluar el comportamiento del modelo económico implantado a partir de 1989 para justipreciar el cumplimiento de los seis puntos establecidos en el acuerdo, es una ardua tarea en la que ya hay investigadores especializados trabajando y con algunos estudios sectoriales concluidos ${ }^{3}$.En ellos nos hemos apoyado para hacer nuestra propia valoración, utilizando las percepciones del demos y algunos parámetros económicos que permitan establecer una cierta medida de la realidad del entorno interno, pues es sabido que los indicadores son solo una herramienta empleada en el esfuerzo por sintetizar la complejidad de una sociedad.
La desarticulación del Foro Económico permitió manos libres al gobierno para imponer su interpretación al cumplimiento de los lineamientos trazados, cuando ya tenía en marcha su reforma económico neoliberal, ${ }^{4}$ imbuida de una alta dosis de triunfalismo ante los rápidos éxitos relativos obtenidos al inicio, medidos-entre otras variables- a través de la tasa de crecimiento económico promedio, que en el quinquenio 89-94 alcanzó el 5.9\%. Apoyó ese triunfalismo el colapso del sistema socialista, con la consecuente unipolaridad sostenida sobre la tesis de que el capitalismo a esas alturas ya globalizado- era la única alternativa viable y el neoliberalismo su mejor modelo, de ahí la frase de Bush padre: "Nuestro estilo de vida no es negociable", cautivado por el crecimiento económico de su país. Pero para el 2009. el debilitamiento del sistema era evidente y se encontraba en franca desaceleración, tal como lo mostraba el indicador de volumen de la actividad económica (Ivae), ${ }^{5}$ publicado periódicamente por el Banco Central de Reserva (BCR), según el cual, a inicios del 2004 era de aproximadamente el $2 \%$, tuvo su mayor crecimiento entre octubre de 2005 y enero de 2006 cuando alcanzó una cifra bastante arriba del $5 \%$, y comenzó a caer a partir de entonces hasta llegar a un valor negativo de $-3 \%$ en enero de 2009. Para completar el panorama macroeconómico tan desalentador llegó la crisis mundial que, si bien es cierto, la recibimos con la tasa de inflación más baja de la región (5.5\% a diciembre de 2008)، también lo era su manifiesta tendencia a aumentar ${ }^{6}$ y la desaceleración del crecimiento económico:

\begin{tabular}{|c|c|c|c|c|c|}
\hline Período & $89-2004$ & $95-99$ & $99-04$ & $04-08$ & Ene 08-09 \\
\hline Tasa (\%) & 5.9 & 3.9 & 2.1 & 4 & -2.9 \\
\hline
\end{tabular}

(Elaboración propia con base en datos del BCR; tasa promedio por período)

\footnotetext{
3 Para una mayor información, que mejore la visión elaborada por la investigación, se recomienda al lector la obra del Licenciado Juan Héctor Vidal, titulada "de la ilusión al desencanto" publicada por nuestra Universidad.

4 Las políticas de desregulación económica, liberalización comercial y privatizaciones fueron los ejes estratégicos del modelo y respondían a las propuestas del "Consenso de Washington".

5 Este es un indicador de coyuntura que calcula el comportamiento de la producción a precios constantes, tomando un precio base aplicado a una muestra que comprende sectores, productos y un número significativo de empresas; en el caso de El Salvador, los precios de referencia para el Sistema de Cuentas Nacionales son los de 1990.

6 Según datos del BCR, en el 2006 la tasa de inflación fue del $3.4 \%$ y en el 2007 un poco superior al $4 \%$.
} 
Al fomentarse un estilo de vida basado en las apariencias o, como dicen algunos, con la mentalidad consumista de países desarrollados en un país subdesarrollado, la brecha comercial al 2007 había alcanzado una cifra cercana a los \$ 4.700 Millones, con una propensión a seguir creciendo.7 Indudablemente que los motores de la economía se venían debilitando progresivamente, en especial la agricultura, que prácticamente fue abandonada a su suerte dentro del modelo, bajo el criterio mercantilista de que era más rentable importar que cultivar, e impulsar la maquila para compensar la pérdida de fuentes de trabajo generadas en el campo y a la vez elevar las exportaciones. Los indicadores macroeconómicos muestran cómo el nivel general de la economía se comprimía, la inflación aumentaba y la tasa de desempleo nacional crecía del $6.8 \%$ en el 2004 al $7.2 \%$ en el
2007, a lo que debemos agregar el nivel de subempleo que, según datos del PNUD (2010), en el 2007 alcanzó la cifra del $43 \%{ }^{8}$

Las percepciones que la colectividad ha venido incubando en su constructo mental desde la vivencia diaria, independientemente de los principios teóricos de la economía clásica y de su mayor o menor conocimiento de los datos macroeconómicos, es el otro elemento para hacer el análisis; según la encuesta Mitofsky de mayo 2010, cuando el cuarto período de gobiernos de Arena estaba por concluir, el $84 \%$ de la población consideraba que la economía estaba peor y solo el $13 \%$ decía que mejor; pero más importante era la tendencia a través de varias mediciones realizadas por la misma empresa encuestadora:

\begin{tabular}{|l|c|c|c|c|c|c|c|c|c|}
\hline \multicolumn{1}{|c|}{ Mes } & May08 & Ago-08 & Nov-08 & Feb-09 & May-09 & Ago-09 & Nov-09 & Feb-10 & May-10 \\
\hline Mejor & 8 & 11 & 11 & 14 & 10 & 17 & 22 & 20 & 13 \\
\hline Peor & 91 & 88 & 88 & 85 & 89 & 82 & 77 & 78 & 84 \\
\hline
\end{tabular}

(Datos en porcentaje de respuestas, tomados de la encuesta Mitofsky "Evaluación de gestión")

El pueblo tiene conciencia de que el modelo neoliberal no ha satisfecho las demandas de la mayoría de la población y, por ende, ha dejado de cumplir con lo mandatado constitucionalmente respecto al compromiso de garantizar el goce y el respeto universal y efectivo de los derechos y libertades humanas, transgrediendo lo establecido en el acuerdo de paz; esta percepción inicial se sigue conformando a la luz de los resultados obtenidos por nuestra Universidad, a través del Centro de Investigaciones de la Opinión Pública Salvadoreña (Ciops). En su estudio del 2008 se evidenció que los tres problemas que más afectaban en ese momento a la población eran: a) todo más caro $(66.9 \%), b)$ el desempleo (57.5\%) y c) La delincuencia (23.3\%); además, casi ocho de cada diez encuestados opinaban que en los meses que faltaban para concluir el período del ex presidente Saca, la economía no mejoraría.

En este segundo momento del análisis, las referencias anteriores nos demuestran un desmejoramiento en la calidad de vida de la mayoría de la población salvadoreña e inducen hacia la causa fundamental que explica la tendencia cada vez más intensa del flujo migratorio en busca de las

\footnotetext{
${ }^{7}$ En la obra del licenciado Juan Héctor Vidal, se afirma que la brecha comercial pasó de casi $6 \%$ en 1990 al 25\% en el 2008 en relación al PIB, a causa de la precaria situación de la producción nacional ante la competencia que generan las importaciones, impactando sobre todo a los bienes de consumo, rubro en el que la brecha pasó del $7 \%$ al $13.4 \%$ entre 1991 y 2008.

${ }^{8}$ Se considera población subempleada toda aquella cuyo ingreso es menor al salario mínimo.
} 
oportunidades que el país no ofrece, a pesar de los riesgos implicados en tal aventura, el incremento de medidas en el país receptor para evitar dicho flujo y la persecución interna para deportarlos. Con la crisis, las remesas familiares venían teniendo una tendencia decreciente, aumentando con ello los problemas de las familias que dependían de ese ingreso para su subsistencia. En el primer trimestre del 2009 se reporta una reducción del 7.5\%, en relación con el mismo período de 2008, año en el que dicho flujo de divisas llegó a representar el $17 \%$ del PIB. Algo más, este concepto de "mayoría de la población" incluye ahora a un amplio sector de la identificada como clase media, que cada vez ve acrecentar sus dificultades económicas, tanto a causa del desempleo como del costo de la vida, y coincide con los otros estratos en percibir un complicado escenario económico, forzándolo a buscar nuevos horizontes, aunque generalmente en condiciones migratorias diferentes. Actualmente se estima que han emigrado hacia los Estados Unidos un aproximado de 2.5 millones de compatriotas.

Es sabido que la democracia excluyente y la concentración de la tierra en pocas manos son causas de nuestra violencia estructural; el haber trastocado el espíritu de la propuesta general del acuerdo para reformar el agro nos está pasando ahora la factura:

- La macroeconomía se ha venido vanagloriando de mantener la tasa de inflación más baja de Centroamérica, pero según datos del estudio del PNUD (2010, p.26), la economía familiar ha visto aumentar el valor de su canasta básica alimenticia urbana en casi 20\% en 2009 con relación al 2007, y la rural en aproximadamente en $16 \%$.
- Otra información del referido estudio nos dice que "Ya entre el 2006 y 2007, el porcentaje nacional de hogares pobres había aumentado de $30.7 \%$ a $34.5 \%$. En las zonas urbanas la pobreza pasó de $27.7 \%$ a $29.8 \%$, mientras que en las rurales, de $35.8 \%$ a $43.8 \%$.

- La necesidad de importar bienes de consumo primario ha dejado los precios de mercado a los vaivenes de situaciones exógenas.

El epílogo de la lectura, a partir de los exiguos datos expuestos, es que la deuda histórica estatal en cumplir con los derechos económicos, sociales y culturales se ha acrecentado, poniendo en riesgo la gobernabilidad democrática del país ante la posibilidad del desbordamiento de las protestas mediante acciones políticas, reclamando por una mejor calidad de vida en temas tan sensibles como la salud, el trabajo, el agua, la vivienda, el costo de la alimentación básica, la seguridad, el transporte, la educación y la vulnerabilidad medioambiental.

\section{CONCLUSIONES Y RECOMENDACIONES}

La legitimidad del acuerdo de paz, así como la pertinencia de sus objetivos, ${ }^{9}$ es resultado de la necesidad por resolver la confrontación bélica imperante durante 12 años, a pesar de que el haberse abrogado los miembros de ambas comisiones la representación de todos los salvadoreños para tomar las decisiones, es considerado por algunos de los entrevistados como una debilidad del pacto, con el argumento de no haberse sentido realmente representados. Para nosotros, la estructuración de la mesa de diálogo-negociación

\footnotetext{
${ }^{9}$ Los objetivos fueron enunciados en el Acuerdo de Ginebra: 1) Terminar con el conflicto armado por la vía política al más corto plazo posible. 2) Impulsar la democratización del país. 3) Garantizar el irrestricto respeto a los derechos humanos. 4) Reunificar la sociedad salvadoreña.
} 
era comprensible por las circunstancias precedentes, y por tratarse de una solución esencialmente política, que jurídicamente bien puede dársele el carácter de una constituyente, por las reformas realizadas al orden jurídico primario o fundamental, e incluso, nos atrevemos a afirmar que-al menos sectorialmente-mejor conformada que las anteriores en cuanto a su estructuración. Sin embargo, el no habernos asegurado la intervención democrática más allá de la actividad electoral, es una inicial deuda histórica del nuevo contrato social.

Desde el punto de vista filosófico, consideramos que siempre estuvo presente la finalidad de lograr el bien común, y la jurídica de establecer un orden permisible para que cada persona pudiese desempeñar su potestad libertaria como ser individual, y al mismo tiempo, ser miembro de una colectividad; el rescate de nuestra memoria histórica nos proporciona el conocimiento suficiente para afirmar que esos ideales han sido tradicionalmente empleados para recubrir las verdaderas intenciones de las decisiones tomadas por los grupos de poder, siempre orientadas a favorecer sus particulares e inmediatos intereses, al margen de las repercusiones en el largo plazo. He aquí un hallazgo del estudio: los intereses del bloque dominante en ningún momento de la negociación se vieron realmente afectados. Al recuperar en 1989 el control del Ejecutivo, tuvieron el tiempo suficiente para ir desvirtuando la reforma agraria en defensa de sus intereses y reconciliarse con la Fuerza Armada luego de haberla utilizado como medio de negociación; ambas acciones fueron legalmente justificadas cumpliendo el debido proceso, pero ni legítimas, ni efectivas para cumplir con los objetivos del acuerdo referidos al irrestricto respeto a los derechos humanos y a la reunificación de la sociedad salvadoreña.
El Salvador del presente constituye un enorme laboratorio mental para aplicar la ambigüedad y la paradoja, ante los desafíos de la necesidad de la descentralización frente al histórico verticalismo del poder centralizado; del estigma de una añeja democracia excluyente y la exigencia de una sociedad organizada para participar en las decisiones trascendentales de su incumbencia; del requisito de una gobernabilidad democrática para lograr los objetivos del milenio y su debilitamiento, que obliga al uso los elementos materiales e ideológicos del poder para mantener una precaria legitimidad; de la primacía de los intereses particulares ante las penurias de la colectividad; de la cultura del individualismo confrontada con la solidaridad necesaria para pasar del caos al orden mediante la cooperación orgánica entre el gobierno y sus gobernados, ambigüedad y paradoja esta, que hace recordar la célebre frase de John Fitzgerald Kennedy cuando asumió la presidencia de su país: “No preguntes lo que tu país puede hacer por ti, pregunta lo que tú puedes hacer por tu país." Sentenciando después: "Finalmente, tanto si son ustedes ciudadanos de los Estados Unidos como si lo son del mundo, exijan de nosotros la misma generosidad de fuerza y sacrificio que nosotros les pedimos a ustedes".

A partir del inédito suceso con la llegada de un gobierno de izquierda a la presidencia del Órgano Ejecutivo en junio de 2009, Ios elementos teóricos precedentes, conjuntamente con las contradicciones de la realidad, se han vuelto aportes fundamentales para enfrentar la crisis heredada. Una primera medida para pasar del discurso a la praxis y comenzar a refundar las bases de una nueva sociedad, es decirle al demos la verdad, este es un compromiso ético si se quiere rescatar la confianza de la población, sustancialmente debilitada ante la negación de lo que se nos decía en los informes a la 
nación y a través de la propaganda, por la cotidianeidad del diario vivir, desde la cual ha sido conformado un imaginario colectivo a partir de la simbiosis entre los discursos y las prácticas sociales, objetivizadas por los datos económicos, y los de las percepciones. La autenticidad de esa verdad se ha de ver reflejada en acciones, comenzando por refundar la democracia y los principios que le son inherentes; por aceptar el fracaso, en el largo plazo, de las políticas económicas implementadas desde 1989 con su secuela de un acrecentamiento en la desigualdad, y por asumirla responsabilidad que tiene el Estado acerca de los hechos de graves violaciones a los derechos humanos incumpliendo los compromisos jurídico-políticos internos e internacionales derivados de su aceptación. Es cada una de esas exigencias el inicio necesario, a lo mejor no suficiente, para diseñar las políticas públicas encaminadas hacia el logro de una sociedad con un tejido social funcionando armónicamente a partir de una gobernabilidad democrática.

El hacer que todos los sectores sociales y políticos aceptemos como guía permanente de nuestras acciones las reglas del juego establecidas en nuestra constitución, es otro escollo a superar, de suyo extremadamente complicado si se toma en cuenta la perniciosa actitud de anteponer lo político a lo jurídico. El retorno a los principios filosóficopolíticos del pacto social de 1992 ha de permitir crear el escenario adecuado para iral reencuentro de la senda de la justicia y la validación práctica de los derechos humanos; pero hemos de tener cuidado esta vez de mantener activa nuestra memoria histórica para no repetir los errores del pasado, entre ellos el excesivo optimismo que a muchos nos contagió en aquella oportunidad, sin tomar en cuenta toda la experiencia vivida desde la invasión española hasta nuestros días, con su estela de tradiciones, paradigmas, valores y visiones ideológicas que han venido condicionando la forma de organizarnos estructuralmente, convirtiendo el cambio social en un movimiento pendular de esperanza y desencanto, de embeleso y frustración.

\section{REFERENCIAS}

- Acuerdos de Paz, El Salvador (2007), Publicación con motivo de conmemorarse el XV aniversario de la firma del Acuerdo de 1992, San Salvador.

- Constitución de la República de El Salvador 1983.

- Declaración Universal de los Derechos Humanos (1948).

- PNUD (2009) El Salvador, el camino hacia un nuevo ciclo político: escenarios de gobernabilidad 2009-2010, San Salvador. El Salvador.

- CEPAL (2008). Informe regional sobre Desarrollo Humano para América Latina y el Caribe. San José, Costa Rica.

- BCR, informes periódicos sobre la economía del país EI Salvador.

- MITOFSKY, encuesta de opinión 2010, "evaluación de gestión"

CIOPS, encuesta de opinión 2008. Universidad Tecnológica de El Salvador.

- VIDAL, Juan H., (2010) De la ilusión al desencanto. Tecnoimpresos. 